\title{
DINÁMICA DE LOS FRENTES EN OTOÑO A SU PASO POR EL MERIDIANO 0 ${ }^{\circ}$ EN EUROPA OCCIDENTAL
}

\author{
Ma Carmen Moreno García \\ Miquel Puig i Trens
}

\section{RESUMEN}

Se analiza la frecuencia de paso de frentes durante los meses de octubre, noviembre y diciembre del período 1980-1996, a lo largo del meridiano $0^{\circ}$ de Greenwich, entre los $25^{\circ}$ y $\operatorname{los} 70^{\circ}$ de latitud norte. Los frentes son analizados por tipos - fríos, cálidos y ocluidos - y según la latitud - una vez dividido el meridiano principal en sectores de $5^{\circ}$ de latitud-. Por último, se fija la atención especialmente en el sector latitudinal $40^{\circ}-45^{\circ} \mathrm{N}$ del meridiano, a su paso por el territorio peninsular, sobre el cuadrante nororiental.

Palabras clave: frente, meridiano $0^{\circ}$, latitud, otoño, cuadrante nororiental de la Península Ibérica.

\section{ABSTRACT}

One must analyse the frequency of the meteorological fronts during october, november and december of the period 1980-96 along Greenwich meridian between $25^{\circ}$ and $70^{\circ}$ of north latitude. The fronts are analysed as per cold, warm and occluded, and according to the latitude once the main meridian has been divided in sectors of $5^{\circ}$ of latitude. Lastly, attention must especially be focused in the latitudinal sector $40^{\circ}-45^{\circ} \mathrm{N}$ of the meridian which crosses the peninsular territory in the north-east quadrant.

Key words: front, meridian $0^{\circ}$, latitude, autumn, north-east quadrant of the Iberian peninsula.

\section{RÉSUMÉ}

On analyse la fréquence du passage de fronts pendant les mois d'octobre, novembre et décembre du période 1980-96 sur la ligne du méridien $0^{\circ}$ ou de Greenwich entre $25^{\circ}$ et $70^{\circ}$ de latitude nord. Les fronts sont analyzés par types - froids, chauds et occlus - et selon la latitude - ayant divisé le méridien principal en différents secteurs de $5^{\circ}$ de latitude - . On fixe l'attention aussi spécialment sur le territoire péninsulaire, dans le quadrant nordoriental.

Mots clés: front, méridien $0^{\circ}$, latitude, automne, quadrant nordoriental péninsule Ibérique. 


\section{Introducción}

El estudio llevado a cabo en este trabajo consiste en la realización de un seguimiento del comportamiento de los frentes en la antesala de la Europa occidental, concretamente sobre el meridiano cero, que atraviesa parte del territorio peninsular español. Se va a contabilizar el número de frentes que cruzan dicho meridiano, así como su tipo, y se analizarán las características globales de dichos frentes, a escala temporal anual en mayor medida, y mensual. La escala espacial utilizada es la sinóptica dado que el mayor volumen de información acerca de los frentes viene a esta escala.

Como es bien sabido que la circulación general dominante en la atmósfera en latitudes templadas es, o bien $\mathrm{W}$, o NW, o SW, este meridiano será representativo para conocer cuántos y qué tipos de frentes en otoño son los más usuales al adentrarse en Europa Occidental según la latitud.

Resulta interesante el hecho que pase por ciudades como Londres, o cerca de Le Havre, Zaragoza, Lleida, Castellón, y relativamente cerca de ciudades tan importantes también como París o Barcelona, éstas ya algo más desplazadas. Asimismo el hecho de que atraviese buena parte de la Península, en el cuadrante nororiental, le confiere otro motivo de interés.

Por otra parte no hay demasiados estudios realizados en nuestro país y referidos al ámbito peninsular, donde los frentes sean el objeto de análisis preferente, de carácter climático. Uno de los pocos conocidos y que constituirá un modelo de referencia es el de MARTÍN VIDE (1987), acerca de la frecuencia de paso de los frentes en el litoral mediterráneo español.

\section{Datos de origen}

La información ha sido obtenida a partir del Boletín Meteorológico Diario, publicado por el INM del Ministerio de Medio Ambiente. Los mapas analizados han sido los de superficie a las $6 \mathrm{~h}, 12 \mathrm{~h}$ y $18 \mathrm{~h}$ de los meses de octubre, noviembre y diciembre - corresponde grosso modo al otoño - desde el año 1980 hasta 1996. Con estos mapas lo que se ha llevado a cabo ha sido la contabilización de los frentes, tanto fríos, cálidos, como ocluidos, a partir de la observación de estos tres mapas diarios. Además se ha realizado un seguimiento de cada frente según la latitud por la que viajaban aprovechando las divisiones de los paralelos en intervalos de $5^{\circ}$ en el meridiano cero o de Greenwich. Así, el meridiano está dividido en $5^{\circ}$ desde la latitud $25^{\circ}$, hasta la latitud $70^{\circ}$-la proyección utilizada anterior a 1986 sólo permitía analizar hasta la latitud $65^{\circ}-$. De este modo se puede hacer un seguimiento detallado del comportamiento más habitual de los frentes.

La escasa diferencia horaria entre dos mapas consecutivos permite hacer un seguimiento sin apenas problemas, del camino seguido por cada uno de los frentes a pesar del salto que se produce al tratar con información discreta como son los mapas, y no continua. Las dificultades han aparecido en el momento que en algún caso algunos mapas no han podido ser localizados, o se han extraviado. De los 1564 días analizados, en 51 casos se ha dado este inconveniente lo que ha supuesto un 3,2\% de error respecto al total de mapas analizados. Es un error poco importante y no debe alterar de forma significativa los resultados obtenidos. Otro problema aparecido es el hecho de que en los mapas sinópticos realizados durante estos 17 años puede haber variado el criterio de hasta dónde dibujar la traza 
de cada uno de los frentes, puesto que, probablemente, dada la amplitud del período haya habido más de una persona encargada de realizar los mapas. Hay que tenerlo presente, ya que a la escala que trabajan estos mapas -1:40.000.000 - un error en la traza de unos milímetros puede ser significativo.

\section{Características generales de los frentes}

Un frente constituye la superficie límite que separa masas de aire diferentes. Estas masas de aire presentan distintas características termodinámicas; dichas características vienen dadas por el lugar donde se generan las masas de aire - regiones manantiales $o$ fuente - así como por los lugares que atraviesan a lo largo de trayectorias que pueden llegar a miles de kilómetros.

A través del movimiento de las masas de aire se produce un intercambio de energía entre las zonas ecuatoriales, las cuales presentan un exceso de energía interna, y las altas latitudes, que presentan un claro déficit.

Los frentes o fronteras de separación entre masas de aire distintas, objeto de estudio en este trabajo, permiten el intercambio de energía en forma de calor a partir de la nubosidad y la precipitación - condensación-, entre las masas de aire.

El aire caliente, al enfriarse al entrar en contacto con la masa de aire fría, produce una liberación de la energía almacenada del agua en forma de vapor, en la masa de aire cálida, a partir del proceso de la condensación. Esta liberación de energía calorífica conocida como calor latente, podrá ser utilizada por el frente al poderse favorecer los movimientos verticales gracias a la transformación de la energía calorífica en cinética. Esta transformación favorecerá a su vez la actividad del frente, sobre todo, si el aporte lleva consigo humedad. La actividad de un frente también vendrá dada entre otros factores, no sólo por las características de las masas de aire, tales como las diferencias de energía interna entre ambas, las condiciones en altura, etc., sino también por el movimiento relativo entre ellas. Así, una masa de aire que empuje perpendicularmente a otra tendrá un frente con mayor rendimiento que si el empuje se realiza a modo de cizalla entre ambas masas. Con este último movimiento, que tenderá a ser paralelo, obtendremos un frente más o menos estacionario, mucho menos activo que en el primer caso como es, por ejemplo, una configuración isobárica en forma de vaguada.

\section{Frecuencia en otoño de los frentes (serie 1980-1996)}

A lo largo del período estudiado de 17 años, cruzaron el meridiano cero 2.210 frentes entre los $25^{\circ}$ y $70^{\circ}$ de latitud. De este dato general se desprende que cada otoño aproximadamente pasan unos 130 frentes de media. Como es sabido, el trimestre estudiado tiene 92 días con lo que cada día pasan 1,4 frentes, es decir, entre uno y dos frentes, en promedio. La desviación estándar es de 13,1 con lo que el 68,3\% de los años el número de frentes está comprendido entre 116,9 y 143,1 .

De los 2.210 frentes, 941 fueron frentes fríos, 820, cálidos, y 449, ocluidos. Así, el número medio de frentes fríos que cruzan el meridiano cada otoño es de 55,4 , con una desviación estándar de 5,5. Por su parte, la media de cálidos es de 48,2 con una desviación estándar de 6 , mientras que los ocluidos arrojan una media de 26,4 y una desviación de 5,3 frentes. Con esto: cada 1,6 días pasa uno frío; cada 1,9 días pasa uno cálido y cada 3,4 uno ocluido. 


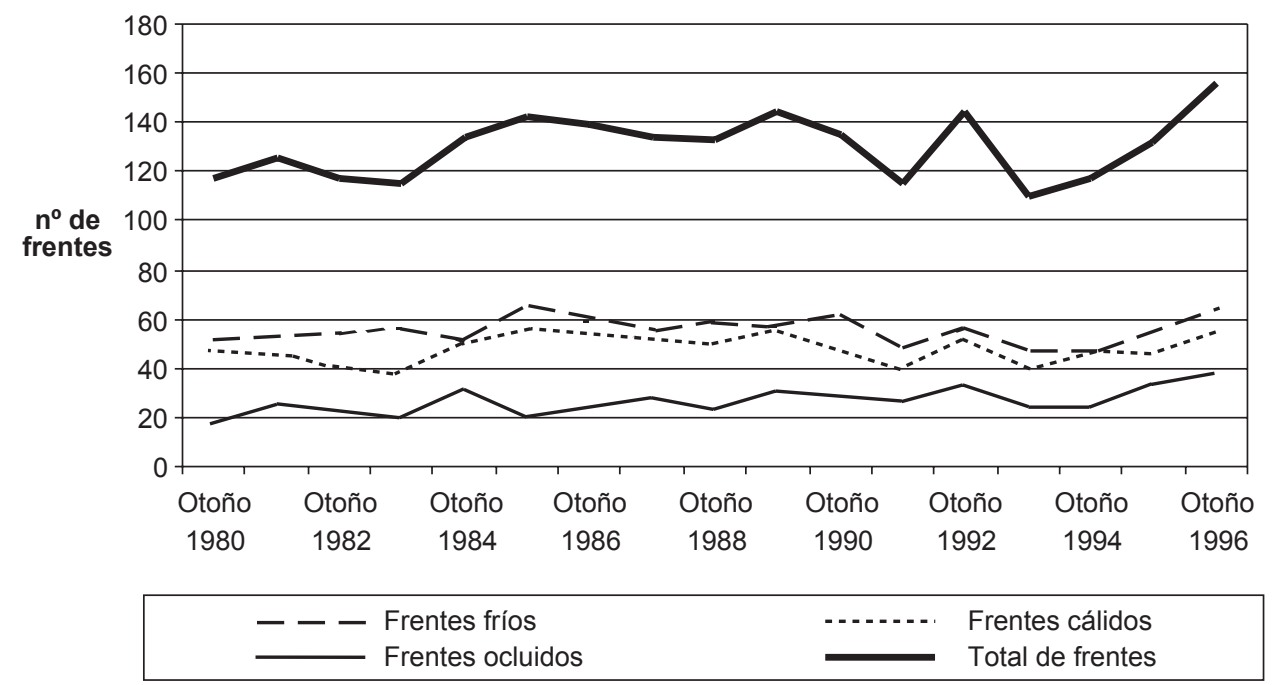

GRÁFICO 1: Curvas correspondientes al $\mathrm{n}^{\circ}$ de frentes, totales y por tipos, en cada otoño de los años 1980-1996.

En el gráfico 1 se puede observar que los totales de cada uno de los otoños varían año tras año, siendo 1996, el último año de la serie, el que presenta el mayor número de frentes cruzando el meridiano.

En todos los casos se observa que la mayor frecuencia de frentes corresponde a los de carácter frío, seguidos por los de carácter cálido, y por último, los ocluidos con una frecuencia bastante menor que los dos primeros. Esto se debe al hecho de que los frentes fríos en muchos casos se presentan aisladamente, mientras que los otros dos tipos van acompañados generalmente del frente frío. Además, los frentes ocluidos cruzan con menor frecuencia el meridiano, porque en muchos casos, la oclusión de la borrasca asociada se produce una vez ésta ha superado el meridiano y se ha adentrado en Europa.

En el cuadro I se recoge el $\mathrm{n}^{\circ}$ de frentes contabilizados cada otoño clasificados por tipos. Hay que hacer notar que desde el año 1980 hasta el 1985 inclusive, no se han podido contar los frentes sobre la latitud $65^{\circ}-70^{\circ}$ ya que los mapas del Boletín en esa época no la incluían.

\section{Frecuencia en otoño según la latitud}

En este apartado se analizará la frecuencia y distribución de los frentes por los distintos sectores latitudinales anteriormente mencionados. No hay que caer en el error de confundir el número de frentes con los sectores ocupados por éstos dado que esta última cifra será mayor lógicamente.

En lo referente a la frecuencia, los 2.210 frentes han ocupado a lo largo de la serie de 17 otoños, 4.736 sectores latitudinales. De este dato obtenemos que el número medio de sectores ocupados por cada frente es algo superior a 2 , es decir, unos $10^{\circ}$ de latitud, 
Cuadro I

TOTALES ABSOLUTOS DE FRENTES Y POR TIPOS EN EL OTOÑO DE CADA UNO DE LOS ANOS

\begin{tabular}{|c|c|c|c|c|}
\hline Año & Fríos & Cálidos & Ocluidos & Totales \\
\hline 1980 & 52 & 47 & 18 & 117 \\
1981 & 54 & 47 & 25 & 126 \\
1982 & 54 & 40 & 23 & 117 \\
1983 & 57 & 38 & 20 & 115 \\
1984 & 51 & 52 & 31 & 134 \\
1985 & 65 & 56 & 21 & 142 \\
1986 & 60 & 54 & 25 & 139 \\
1987 & 55 & 52 & 27 & 134 \\
1988 & 60 & 50 & 23 & 133 \\
1989 & 58 & 56 & 30 & 144 \\
1990 & 61 & 47 & 27 & 135 \\
1991 & 48 & 41 & 26 & 115 \\
1992 & 57 & 53 & 34 & 144 \\
1993 & 47 & 39 & 24 & 110 \\
1994 & 46 & 47 & 24 & 117 \\
1995 & 53 & 46 & 33 & 132 \\
1996 & 63 & 55 & 38 & 156 \\
\hline
\end{tabular}

aproximadamente. Éste no obstante es un dato general que no da una idea precisa de la longitud de los frentes. Para ello, se analizarán los datos según el carácter de cada frente.

Los frentes fríos ocupan un promedio de 2,6 sectores o unos $12^{\circ}$ ó $13^{\circ}$ de latitud, los frentes cálidos, 1,8 , mientras que los ocluidos 1,9 sectores o $10^{\circ}$ de latitud, aproximadamente. Resulta de interés observar el hecho de que los frentes cálidos y los ocluidos tienen prácticamente la misma longitud. Esto se debe con toda probabilidad a que la borrasca asociada a los sistemas frontales, al madurar, transforma el carácter del frente cálido en ocluido. Esto tiene lugar cuando el frente frío, detrás del cálido, al llevar más velocidad que este último obliga a la masa cálida a ascender hacia niveles superiores de la atmósfera. Este proceso de oclusión se lleva a cabo siempre desde el punto más septentrional al más meridional del sistema frontal, en función de la longitud del frente cálido. De este modo se entiende fácilmente que las longitudes del frente cálido y ocluido hayan de ser iguales de modo aproximado. La mínima diferencia a favor de la longitud de los ocluidos respecto de los cálidos $-1,9$ y 1,8, respectivamente- pudiera deberse a que los sistemas frontales con frentes cálidos más largos, se ocluyan antes de llegar al meridiano y adentrarse en Europa, mientras que los más cortos lo hagan después de cruzar el meridiano, una vez ya en el continente europeo. Esta hipótesis precisaría de un análisis más riguroso y exhaustivo, que escapa al objeto de estudio de este trabajo. 
Cuadro II

PORCENTAJE DEL N $N^{\circ}$ DE DÍAS CON PASO DE FRENTES, TOTALES O POR TIPOS, EN OTOÑO POR LOS DISTINTOS SECTORES LATITUDINALES SOBRE EL MERIDIANO $0^{\circ}$

\begin{tabular}{|c|c|c|c|c|c|}
\hline Días con... & Frentes (\%) & Fríos (\%) & Cálidos (\%) & Ocluidos (\%) & Sin frentes (\%) \\
\hline $65^{\circ}-70^{\circ}$ & 28,95 & 09,88 & 08,10 & 10,97 & 71,05 \\
$60^{\circ}-65^{\circ}$ & 47,70 & 17,84 & 14,32 & 15,54 & 52,30 \\
$55^{\circ}-60^{\circ}$ & 60,36 & 26,28 & 21,16 & 12,92 & 39,64 \\
$50^{\circ}-55^{\circ}$ & 62,41 & 31,59 & 21,74 & 09,08 & 37,59 \\
$45^{\circ}-50^{\circ}$ & 52,18 & 28,97 & 17,65 & 05,56 & 47,82 \\
$40^{\circ}-45^{\circ}$ & 36,26 & 22.00 & 10,55 & 03,71 & 63,74 \\
$35^{\circ}-40^{\circ}$ & 19,38 & 15,03 & 03,58 & 00,77 & 80,62 \\
$30^{\circ}-35^{\circ}$ & 05,69 & 05,37 & 00,26 & 00,06 & 94,31 \\
$25^{\circ}-30^{\circ}$ & 00,12 & 00,12 & 00,00 & 00,00 & 99,88 \\
\hline
\end{tabular}

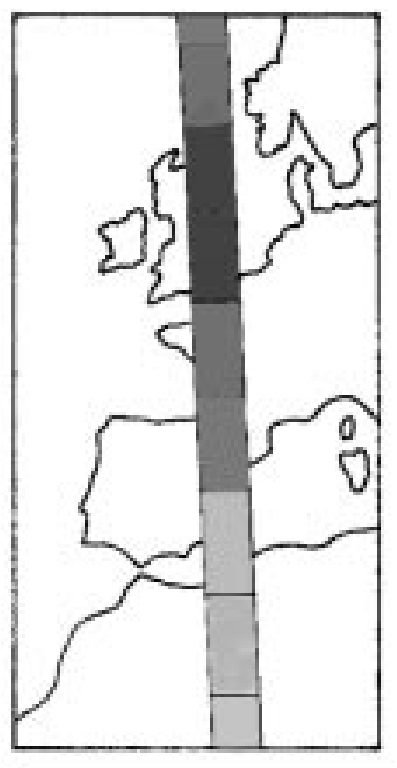

MAPA 1: Porcentaje del $\mathrm{n}^{\circ}$ de días con paso de frentes totales en otoño por los diferentes sectores latitudinales del meridiano $0^{\circ}$. 
La distribución de los frentes según la división de los sectores latitudinales presenta un pico de concentración en la frecuencia del paso de los frentes sobre la latitud $50^{\circ}-55^{\circ}$, siendo ésta de 976 pasos (gráfico 2). De este modo se puede afirmar que de los 1.564 días analizados, en un 62,4\% pasó un frente por dicha latitud. Si nos alejamos de este pico la frecuencia va bajando de forma progresiva y considerable. Más allá de los $35^{\circ}$ y $70^{\circ}$ de latitud el paso de frentes se convierte en algo excepcional. Por ejemplo, sobre la latitud $25^{\circ}-30^{\circ}$ en el meridiano cero - sobre el Sahara - sólo han pasado 2 frentes fríos a lo largo de toda la serie estudiada (véase mapa 1). Por encima de $\operatorname{los} 70^{\circ}$ de latitud, la fuente de información analizada no permite el estudio puesto que los mapas sinópticos no van más allá de esa latitud. No obstante, como es bien sabido es de esperar que hacia el polo la frecuencia se reduzca al mínimo. Se analizarán ahora los frentes, no por totales sino por el carácter de ellos.

La distribución de los frentes fríos tiene como máximo de frecuencia de paso sobre el sector de latitud $50^{\circ}-55^{\circ}$. En esta franja latitudinal los frentes fríos han ocupado el sector en 494 ocasiones. Ello supone el 50,6\% de todos los frentes que han pasado por este sector durante 17 años. En todos los sectores, los frentes fríos tienen una mayor presencia que los otros dos tipos de frentes siendo mínima dicha diferencia hacia las latitudes más altas. En cuanto al número de días con un frente frío entre $50^{\circ}-55^{\circ}$, en un $31,5 \%$ de los días hay uno de media, es decir, cada tres días, aproximadamente (cuadro II).

La distribución de los frentes cálidos no presenta sólo un máximo, como en el caso de los de carácter frío, sino dos. Concretamente estos picos se encuentran sobre las latitudes $50^{\circ}-55^{\circ}$ y $55^{\circ}-60^{\circ}$. En estas latitudes, los frentes cálidos han ocupado dichas franjas 340 y 331 ocasiones, respectivamente. De estos datos se desprende que un $34,8 \%$ de todos los frentes, fueron cálidos en la latitud $50^{\circ}-55^{\circ}$, y que un $33,9 \%$ lo fueron entre $55^{\circ}-60^{\circ}$ de latitud. Si se contabilizan los días con frentes cálidos, un $21,7 \%$ y un $21,1 \%$ de los días, respectivamente, pasó un frente cálido, es decir, cada 4 ó 5 días, en promedio.

Los frentes ocluidos presentan en su distribución un máximo desplazado hacia las altas latitudes, sobre $60^{\circ}-65^{\circ}$, siendo la frecuencia de éstos, mayor que la de los frentes cálidos a partir de los $60^{\circ}$. Entre los $60^{\circ}$ y $65^{\circ}$, los frentes ocluidos han ocupado 243 veces esta franja latitudinal, lo que supone un $32,6 \%$ del total de frentes que pasaron por esta latitud. De los

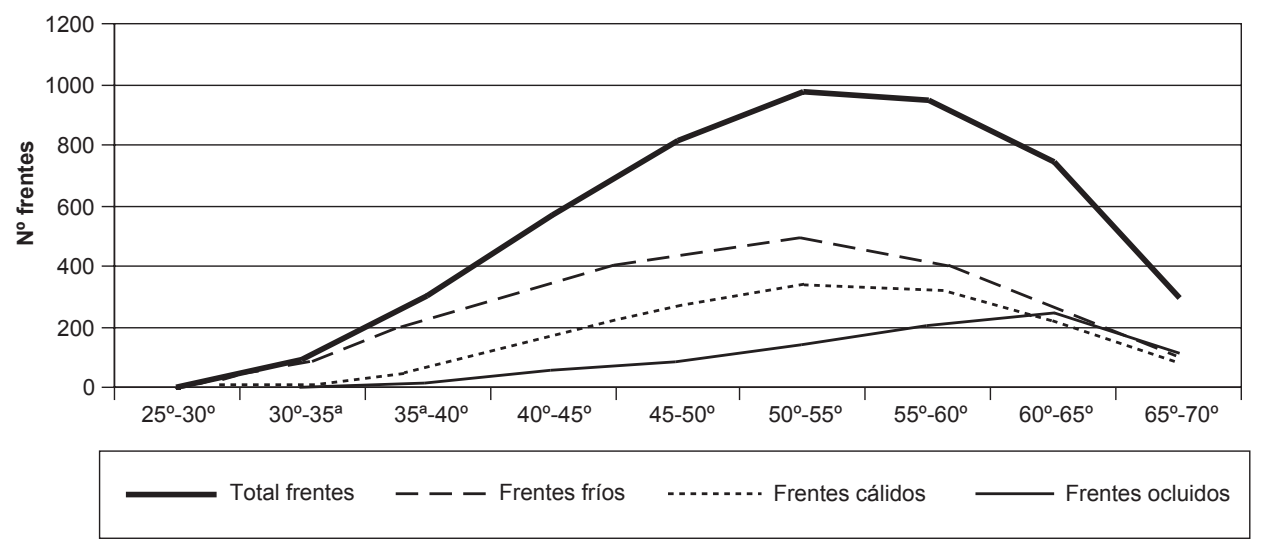

GRÁFICO 2: Curvas correspondientes al $\mathrm{n}^{\mathrm{o}}$ de frentes, totales y por tipos, contabilizados a su paso en otoño por los distintos sectores latitudinales del meridiano $0^{\circ}$. 
1.564 días analizados, en un 15,5\% de los días hubo un frente ocluido, o sea, uno cada 6 ó 7 días de promedio, aproximadamente.

El máximo total de frentes se concentra entre las latitudes $50^{\circ}$ y $60^{\circ}$, algo lógico, ya que es aquí donde se localiza de un modo habitual la discontinuidad que separa el aire frío de las altas latitudes del aire cálido de latitudes subtropicales, o frente polar, del que nacen, precisamente, los frentes.

Si se observa atentamente el gráfico 2 se ve que el grueso de los frentes se va desplazando hacia las latitudes más altas según si se toman en consideración los frentes fríos, cálidos o los ocluidos. Si como anteriormente se ha dicho, las borrascas maduran una vez se han adentrado en el continente, parece lógico pensar el hecho de que el grueso de los frentes ocluidos esté por encima de los $60^{\circ}$ de latitud, dado que por los puntos más septentrionales de los sistemas frontales es por donde empieza la oclusión. Superado el meridiano, la oclusión continúa, dependiendo de su velocidad y de la longitud de los frentes, sobre todo, del cálido.

\section{Frecuencia en otoño del paso de frentes en el sector latitudinal $40^{\circ}-45^{\circ} \mathrm{N}$ (sobre el cua-} drante nororiental de la Península Ibérica)

Fijando la atención preferente en lo que ocurre en nuestro país, se analizará el tramo del meridiano $0^{\circ}$ que cruza la Península Ibérica, concretamente, el sector comprendido entre las latitudes $40^{\circ}-45^{\circ} \mathrm{N}$, que se extiende por el cuadrante nororiental. La frecuencia de paso de frentes durante el otoño de cada uno de los años desde 1980 hasta 1996 por este sector latitudinal queda representada en el gráfico 3.

En él se observa que el otoño que presentó más frentes fue el del año 1996 con 52 frentes, mientras que el que registró el mínimo absoluto fue 1988 con un total de 15.

La media de la serie es de 33,4 frentes por otoño, con una desviación de 10,3, lo que no es nada despreciable. Ello confirma que no se observe ningún tipo de concordancia de un año a otro y tampoco ninguna tendencia clara a lo largo de la serie.

Durante el otoño de 1996, se produce el máximo principal de la serie, sucediendo algo similar a lo acontecido en 1981. Sin embargo en 1996, el paso de borrascas profundas y

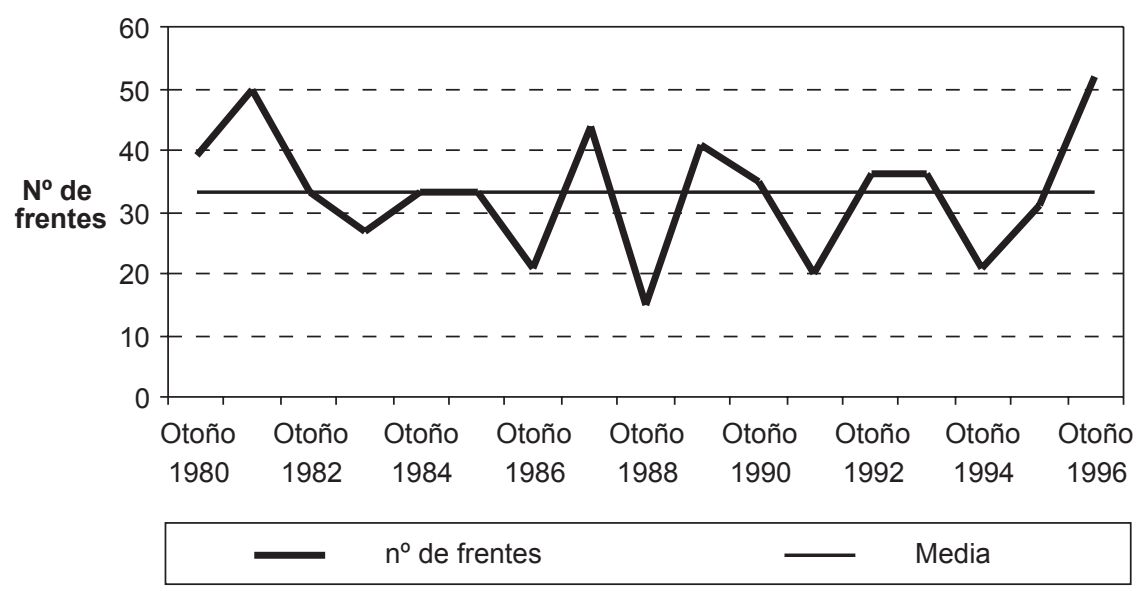

GrÁFICO 3: $\mathrm{N}^{\circ}$ de frentes que pasaron por el cuadrante NE de la Península Ibérica $\left(40^{\circ}-45^{\circ} \mathrm{N}\right)$ entre los otoños de 1980 y 1996. 
frentes ya empezó a mediados de noviembre, unos quince días antes que en 1981. El anticiclón de las Azores adoptó una posición media durante estos quince días estableciéndose una advección del NW que afectaba a la Península Ibérica. Así, el paso de borrascas y frentes por la Península fue continuo. En diciembre esta situación se acentuó todavía más. El anticiclón de las Azores se desplazó en el Atlántico hacia latitudes más septentrionales, mientras que en latitudes saharianas aparecía otro anticiclón. Así, las borrascas sólo ven un camino por el que pasar que es entre ambos anticiclones, por las latitudes que normalmente ocupa el anticiclón de las Azores. Hay que tener en cuenta que los anticiclones se presentan muy móviles y que en sus senos las presiones no son mayores de $1.024 \mathrm{mb}$ en muchos días de este mes. Sólo el anticiclón centroeuropeo a finales de mes se refuerza, pero éste, que ocupa latitudes altas, favorece con su posición el paso de borrascas y frentes sobre el cuadrante noroccidental de la Península Ibérica.

Entre los frentes que pasaron por el cuadrante nordeste de la Península y los que cruzaron el meridiano en toda su extensión hay una cierta correspondencia y ello se debe a que un porcentaje elevado de frentes que cruzaron el meridiano lo hicieron precisamente por las latitudes $40^{\circ}-45^{\circ}$

En 1981 se produce el máximo secundario, con 50 frentes. La causa de este máximo radica en que aunque octubre y noviembre no presentan ninguna anomalía destacable, con una frecuencia en el paso de frentes entorno a la media, en cambio en el mes de diciembre, se produce un aumento importante en el paso de borrascas y de frentes. Ello se debe a que el anticiclón de las Azores se retira muy al sur en el Atlántico, desplazándose toda la circulación general hacia latitudes más meridionales, siendo pues, el paso de borrascas y de frentes por nuestras latitudes mucho más frecuente, similar incluso a lo que ocurre habitualmente en latitudes algo más altas, como las del Frente Polar.

En 1987 y 1989 se observan también frecuencias destacables, por encima de la media. En el primer caso, durante la primera quincena del mes de octubre, la situación predominante fue la entrada de borrascas y vaguadas por el sector norte; en muchos días de ese mes, se advertía la presencia de una borrasca localizada en la mitad sur de la Península Ibérica, aunque no en días continuos. En noviembre también se dio esta última situación. Además, el anticiclón que se sitúa en Europa occidental se desplazó hacia el interior del continente favoreciendo la presencia de borrascas. En el Atlántico, el anticiclón de las Azores permanece lo suficientemente lejos como para ejercer una clara influencia. En diciembre, sobre todo en los primeros quince días, los anticiclones ocuparon regiones muy alejadas del área de estudio, permitiendo el paso sucesivo de frentes por nuestras latitudes.

En el segundo caso, en 1989, es, sobre todo, en noviembre y diciembre, donde se observa algún aspecto significativo. Una vez más, el anticiclón de las Azores se retira hacia el Atlántico, mientras que otro anticiclón se retira hacia Rusia durante varios días, permitiendo la presencia de profundas borrascas al NW de Galicia que van lanzando frentes hacia el área de estudio. Esta situación persiste con más o menos frecuencia durante noviembre e incluso a comienzos de diciembre. En este último mes, el anticiclón Atlántico se desplaza muy al sur del océano, mientras que borrascas muy profundas barren toda Europa, incluyendo la Península Ibérica.

Durante el otoño de 1988 se produce el mínimo absoluto, sólo 15 frentes pasando sobre el área de estudio. A partir del día 20 de octubre, se situó sobre la Península Ibérica un anticiclón que se extendió hacia Europa tomando una posición meridiana, bloqueando el paso de las borrascas. A principios de noviembre, el anticiclón se desplazó hacia Centroeuropa, mientras que una baja se mantuvo durante una semana estacionaria frente a 
Portugal, al W de la Península Ibérica. En la siguiente semana el anticiclón adoptó una posición zonal sobre Europa, evitando el paso de cualquier posible perturbación. Hacia finales de este mes, también, el anticiclón aparece centrado en las Islas Británicas, configurando una advección del NE sobre el cuadrante de estudio, y una borrasca no muy profunda al SW de Portugal. El cambio de mes se manifestó sinópticamente con el anticiclón localizado sobre la Península Ibérica. La segunda y tercera semana de diciembre el anticiclón se situó al norte de Galicia ejerciendo un bloqueo ante las borrascas, y estableciéndose una nueva advección del NE sobre Cataluña. La última semana del año registra, asimismo, el anticiclón, una vez más, sobre la Península para desplazarse posteriormente hacia Centroeuropa. En definitiva, la persistencia de altas presiones en el continente europeo durante este otoño, es la causa que explica la menor cantidad de frentes en el cuadrante nororiental de la Península.

En 1986, 1991 y 1994 se registran los mínimos secundarios, con valores muy similares $-21,20$ y 21, respectivamente-. Durante los diez primeros días de octubre de 1986 se observa la presencia de un anticiclón sobre el Canal de la Mancha; en los días siguientes se desplaza hacia Centroeuropa mientras una borrasca se sitúa al SW de Portugal. La segunda quincena de mes se caracteriza por una situación predominante de dorsal anticiclónica sobre la Península Ibérica. En la primera semana de noviembre vuelve a producirse una advección del NE sobre el cuadrante nororiental de la Península. A la semana siguiente es la presencia de un anticiclón sobre la cuenca mediterránea occidental la que impide el paso de borrascas y frentes. Los últimos diez días muestran un anticiclón sobre Francia que proyecta su influencia sobre las regiones circundantes. En las primeras semanas de diciembre, también domina sobre el Mediterráneo un anticiclón y desde mediados de mes, y ya prácticamente hasta el final, se instalan altas presiones sobre la Península Ibérica.

En el otoño de 1991 destaca la persistencia del anticiclón, primero sobre Francia, y luego desplazándose hacia el NW de Galicia a lo largo de los primeros días del mes de octubre. En la segunda quincena, hay un anticiclón al W de Portugal que en los últimos diez días del mes se sitúa entre Galicia y las Islas Británicas mostrando una posición de bloqueo. Noviembre comienza con un anticiclón sobre la Península; a mediados de mes, la posición de un anticiclón sobre el Mediterráneo siguió manteniendo la estabilidad. Hacia finales del mes el anticiclón abarca prácticamente toda Europa, localizándose algunas borrascas centradas al oeste de Portugal. En diciembre, durante los diez primeros días, un anticiclón de bloqueo se sitúa, asimismo, sobre Europa y una borrasca al oeste de Portugal. Posteriormente, el anticiclón se extiende por toda Europa, desplazándose, incluso, sobre la Península Ibérica.

El primer mes del otoño de 1994 se caracteriza básicamente durante las tres primeras semanas por la gran extensión del anticiclón Centroeuropeo sobre el continente, apareciendo en los últimos días una borrasca sobre las Baleares. Noviembre presenta a mediados de mes el anticiclón sobre la Península Ibérica; en días posteriores subirá de latitud, situándose en Francia y extendiéndose por Europa a finales de mes. En el último mes del año, el anticiclón ocupa las latitudes mediterráneas prácticamente todos los días, localizándose su centro en el Mediterráneo al inicio de mes, sobre la Península a mediados, para desplazarse luego al oeste y finalmente volver a situarse de nuevo sobre la Península.

\section{Conclusiones}

La mayor frecuencia de frentes que cruzan el meridiano $0^{\circ}$ son los de carácter frío, mientras que los ocluidos presentan la menor frecuencia. En el primer caso hay que tener 
en cuenta que muchas veces, aparecen también depresiones o borrascas con un solo frente frío, y en el segundo caso, que el proceso de oclusión se inicia, en muchas ocasiones, una vez que la pareja de frentes (frío y cálido) ya ha cruzado el meridiano.

Los frentes fríos también son los que ocupan en promedio mayor extensión longitudinal, porque abarcan unos $12^{\circ}$ ó $13^{\circ}$ de latitud, mientras que los frentes cálidos y ocluidos, más cortos que los primeros, tienen prácticamente la misma longitud.

La mayor frecuencia de paso de frentes sobre el meridiano $0^{\circ}$ se concentra sobre la latitud $50^{\circ}-55^{\circ}$. En cambio, por debajo de $\operatorname{los} 35^{\circ}$ y por encima de los $65^{\circ}$, los valores se reducen al mínimo. En el caso de los frentes ocluidos su máximo se halla desplazado hacia latitudes más altas $\left(60^{\circ}-65^{\circ}\right)$.

En cuanto a la frecuencia del paso de frentes sobre el sector latitudinal $40^{\circ}-45^{\circ} \mathrm{N}$ del meridiano, que es el que correspondería aproximadamente con el cuadrante nororiental de la Península Ibérica, la media es de 33,4 frentes por otoño.

Los máximos observados en los otoños de 1996, 1981, 1987 y 1989 responden a la debilidad o lejanía de los anticiclones, que dio libertad a las borrascas y frentes, siendo el paso de éstos, pues, más continuado y por latitudes más meridionales. El desplazamiento del anticiclón de las Azores en el Atlántico hacia el sur favoreció la circulación zonal sobre este sector en estudio. Al mismo tiempo, la profundidad de las borrascas y el establecimiento de situaciones sinópticas con gran gradiente bárico contribuyó, en gran medida, a favorecer también la aparición de un mayor número de frentes.

Los mínimos registrados en los otoños de 1988, 1986, 1991 y 1994 obedecen, sobre todo, a la extensión del anticiclón centroeuropeo abarcando gran parte del continente, y a la persistencia de anticiclones de bloqueo sobre la fachada occidental europea (Francia, Islas Británicas) y también sobre la Península. Asimismo, la presencia de borrascas estacionarias no muy profundas al oeste de Portugal, y el establecimiento de advecciones del NE sobre Cataluña, acompañadas en ocasiones, con una baja en la cuenca del Mediterráneo occidental, son causas que han contribuido del mismo modo.

Los valores totales obtenidos en los meses de octubre, noviembre y diciembre son inferiores a los hallados por MARTÍN VIDE (1987) para el litoral mediterráneo español, concretamente, para Valencia, aunque hay que señalar que los períodos analizados no son coincidentes, ya que aquel trabajo se refiere al periodo 1951-60, y éste a uno posterior más reciente. De todos modos, la causa que probablemente mejor pueda explicar esta diferencia la apunta el propio autor en su artículo, cuando se indica que la fuente sinóptica utilizada (en su caso, el boletín francés Bulletin Quotidien d'Etudes) al señalar con gran precisión las trazas de los frentes, prolongadas, muchas veces, hasta sectores meridionales o notablemente alejadas del vórtice de la borrasca ondulatoria a la que van asociados, y representar además todas las superficies de discontinuidad entre masas de aire, por poco relevantes que sean, puede dar lugar a una sobreestimación en el número de frentes, si los comparamos con los obtenidos del análisis a partir de otra fuente sinóptica ${ }^{1}$.

La poca longitud de la serie de otoños analizados impide el determinar ninguna tendencia significativa, aunque al observar el gráfico 3 parezca deducirse una cierta alternancia de un año a otro en los máximos y mínimos, especialmente, en los últimos diez años.

1 MARTÍN VIDE, J. (1987): «La frecuencia de paso de frentes en el litoral Mediterráneo español», Anales de Geografía de la Universidad Complutense, $\mathrm{n}^{\circ}$ 7, pp. 145-153. 